\title{
MERIS-based ocean colour classification with the discrete Forel-Ule scale
}

\author{
M. R. Wernand ${ }^{1}$, A. Hommersom ${ }^{2}$, and H. J. van der Woerd ${ }^{2}$ \\ ${ }^{1}$ Royal Netherlands Institute for Sea Research, Physical Oceanography, Marine Optics and Remote Sensing, P.O. Box 59, \\ 1790AB Den Burg, Texel, the Netherlands \\ ${ }^{2}$ Institute for Environmental Studies (IVM), VU University Amsterdam, De Boelelaan 1087, 1081 HV Amsterdam, \\ the Netherlands
}

Correspondence to: M. Wernand (marcel.wernand@nioz.nl)

Received: 13 July 2012 - Published in Ocean Sci. Discuss.: 31 August 2012

Revised: 27 March 2013 - Accepted: 11 April 2013 - Published: 2 May 2013

\begin{abstract}
Multispectral information from satellite borne ocean colour sensors is at present used to characterize natural waters via the retrieval of concentrations of the three dominant optical constituents; pigments of phytoplankton, non-algal particles and coloured dissolved organic matter. A limitation of this approach is that accurate retrieval of these constituents requires detailed local knowledge of the specific absorption and scattering properties. In addition, the retrieval algorithms generally use only a limited part of the collected spectral information. In this paper we present an additional new algorithm that has the merit of using the full spectral information in the visible domain to characterize natural waters in a simple and globally valid way. This Forel-Ule MERIS (FUME) algorithm converts the normalized multiband reflectance information into a discrete set of numbers using uniform colourimetric functions. The Forel-Ule $(F U)$ scale is a sea colour comparator scale that has been developed to cover all possible natural sea colours, ranging from indigo blue (the open ocean) to brownish-green (coastal water) and even brown (humic-acid dominated) waters. Data using this scale have been collected since the late nineteenth century, and therefore, this algorithm creates the possibility to compare historic ocean colour data with present-day satellite ocean colour observations. The FUME algorithm was tested by transforming a number of MERIS satellite images into Forel-Ule colour index images and comparing in situ observed $F U$ numbers with $F U$ numbers modelled from in situ radiometer measurements. Similar patterns and $F U$ numbers were observed when comparing MERIS ocean colour distribution maps with ground truth Forel-Ule observations.
\end{abstract}

The $F U$ numbers modelled from in situ radiometer measurements showed a good correlation with observed $F U$ numbers ( $R^{2}=0.81$ when full spectra are used and $R^{2}=0.71$ when MERIS bands are used).

\section{Introduction}

The application of optical satellite remote sensing techniques to monitor the radiation scattered back from the water column became a major breakthrough in the seventies for monitoring ocean, sea and coastal areas (IOCCG, 1998). Dedicated ocean colour instruments, like CZCS, SeaWiFS, MERIS and MODIS-AQUA, have provided fundamental new insights into the dynamics and role of oceanic plankton (e.g. Behrenfeld et al., 2006). Observations are now starting to span multiple decades, allowing a first glimpse at long-term variations in the plankton composition of the oceans, which are potentially related to global change (Antoine et al., 2005; Polovina et al., 2008).

With the launch, in 2002, of the MERIS instrument (Rast et al., 1999), which measures water-leaving reflectance in fifteen spectral bands with high signal-to-noise, it became possible to collect water-leaving radiance with high confidence in regional seas and coastal waters. This has led to the development of many new algorithms that can retrieve not only the phytoplankton pigments, but also the mass concentration of suspended material and the absorption by dissolved material (Van der Woerd and Pasterkamp, 2008; Odermat et al., 2012). These algorithms are either simple, calibrated to the 
local water constituents, or complex, with a need for detailed measurements of the specific absorption and scattering properties of these in-water constituents (see, e.g. Tilstone et al., 2012). The derived water-quality parameters are the major products of ocean colour instruments, while the colour itself can be considered as a primary product.

Long before the development of diode arrays to measure spectral radiation, another method had been developed and tested which recorded the colour of natural waters. Towards the end of the nineteenth century, Forel and Ule (Forel, 1890; Ule 1892) proposed a method to classify the colour of the oceans, regional seas and coastal waters using a colour comparator scale. The scale became known as the Forel-Ule $(F U)$ scale and since then scale observations have been performed, generating hundreds of thousands of data points at global scale for more than a century. Recently, it was shown (Wernand and Van der Woerd, 2010a) that the $F U$ scale can be used to characterize the colour of natural waters. More importantly, the analysis of $F U$ colour variation in the North Pacific since 1930 has revealed significant variations at decadal timescales (Wernand and Van der Woerd, 2010b).

In this paper we describe a simple algorithm to couple historically collected ocean colour data, obtained over a long time span, with presently available satellite-derived ocean colour imagery for hindcasting long-term changes. This Forel-Ule to MERIS (FUME) algorithm converts MERIS observations of sea- and ocean colour to chromaticity coordinates and subsequently to a discrete Forel-Ule number. This will result in a new MERIS water quality product that can be used as a simple and straight-forward index of water colour in addition to the water-quality parameters that are retrieved by inversion schemes. Based on the FUME product, ocean colour trends can be constructed, reaching back to over one hundred years. Distinct optical water types can now be classified according to the Forel-Ule scale and this makes it possible to enhance satellite derived products, such as chlorophyll (Moore et al., 2009). Ocean colour remote sensing techniques have traditionally been based on two optical water types, known as "Case 1" and "Case 2" (Morel and Prieur, 1977). However, this classification is mainly based on the intrinsic composition, i.e. the role of algae (and related degradation products) in the generation of water colour.

Moore et al. (2009) proposed extending the optical water classification to eight clusters, based on an unsupervised classification of the NOMAD database of remote sensing reflectance spectra. The reflection spectrum of each satellite pixel has a certain probability of belonging to each of the 8 clusters. Another classification method that can be tuned to local properties is proposed by Hommersom et al. (2011). In this work we go back to use the oldest classification of 21 pre-defined scales and use the relative colour difference (colour comparator scale) instead of absolute remote sensing reflectance to classify each pixel to only one representative $F U$ number.
Table 1. Central wavelengths of the first nine MERIS spectral bands. All bands have a width of $10 \mathrm{~nm}$, with the exception of band $8(7.5 \mathrm{~nm})$.

\begin{tabular}{rrrr}
\hline $\begin{array}{r}\text { MERIS } \\
\text { band }\end{array}$ & $\begin{array}{r}\text { Wavelength } \\
(\mathrm{nm})\end{array}$ & $\begin{array}{r}\text { MERIS } \\
\text { band }\end{array}$ & $\begin{array}{r}\text { Wavelength } \\
(\mathrm{nm})\end{array}$ \\
\hline 1 & 412.5 & 6 & 620 \\
2 & 442.5 & 7 & 665 \\
3 & 490 & 8 & 681.25 \\
4 & 510 & 9 & 708 \\
5 & 560 & & \\
\hline
\end{tabular}

\section{Methods}

In this section we introduce the MERIS satellite data, the algorithm to convert MERIS reflection data to $F U$ numbers and the ship-borne measurements for a first characterization of the FUME results.

\subsection{MERIS products}

MERIS is a $68.5^{\circ}$ field-of-view push-broom imaging spectrometer (Rast et al., 1999) on the ENVISAT platform. It measures the solar radiation reflected by the ocean at a spatial resolution of $260 \mathrm{~m} \times 290 \mathrm{~m}$ in 15 spectral bands. The bands are programmable in width and position, at visible and nearinfrared (NIR) wavelengths. MERIS provides global coverage in 3 days with radiation reflected by the ocean that is atmospherically corrected to derive the normalized waterleaving reflectances, a MERIS Level 2 product (ESA, 2012). The atmospheric correction assumes that the water totally absorbs the NIR, but also includes a correction for those sediment loaded waters where this assumption fails. Normalized water-leaving reflectance (dimensionless) $\left[\rho_{\mathrm{W}}\right]_{\mathrm{N}}$ is defined by Eq. (1) as follows:

$\left[\rho_{\mathrm{W}}\right]_{\mathrm{N}}(\lambda)=\frac{\left[L_{\mathrm{W}}\right]_{\mathrm{N}}(\lambda)}{F_{0}(\lambda)}$,

where $\left[L_{\mathrm{W}}\right]_{\mathrm{N}}$ is the normalized water-leaving radiance (Gordon and Voss, 1999) and $F_{0}$ is the extraterrestrial solar irradiance at wavelength $(\lambda)$. In this analysis, data is limited to the visible spectrum, covering the first nine MERIS bands with bandwidths of $10 \mathrm{~nm}$, except for band 8 which has a bandwidth of $7.5 \mathrm{~nm}$ (Table 1). In the standard processing by ESA, a number of global products are derived together with $\left[\rho_{\mathrm{W}}\right]_{\mathrm{N}}$ that will be used to compare $F U$ products with standard ESA products: Algal- 1 and Algal-2 (the indices for chlorophyll $a$ concentration in Case 1 and Case 2 waters, respectively), SPM (suspended particulate matter) and YS (yellow substance; an index for absorption by dissolved matter). For documentation and additional references, we recommend the MERIS algorithm theoretical baseline document (ESA, 2012). 
Table 2. Chromaticity coordinates $(x, y)$ of the $F U$ scale numbers as determined by Wernand and Van der Woerd (2010a).

\begin{tabular}{lllllllll}
\hline No. & $x$ & $y$ & No. & $x$ & $y$ & No. & $x$ & $y$ \\
\hline FU1 & 0.189 & 0.161 & FU8 & 0.311 & 0.439 & FU15 & 0.410 & 0.478 \\
FU2 & 0.196 & 0.194 & FU9 & 0.337 & 0.463 & $F U 16$ & 0.418 & 0.472 \\
FU3 & 0.213 & 0.255 & FU10 & 0.363 & 0.480 & $F U 17$ & 0.427 & 0.466 \\
FU4 & 0.229 & 0.301 & FU11 & 0.388 & 0.490 & $F U 18$ & 0.440 & 0.458 \\
FU5 & 0.242 & 0.331 & FU12 & 0.394 & 0.488 & $F U 19$ & 0.453 & 0.448 \\
FU6 & 0.263 & 0.373 & FU13 & 0.397 & 0.486 & FU20 & 0.462 & 0.440 \\
FU7 & 0.290 & 0.415 & FU14 & 0.404 & 0.482 & FU21 & 0.473 & 0.429 \\
\hline
\end{tabular}

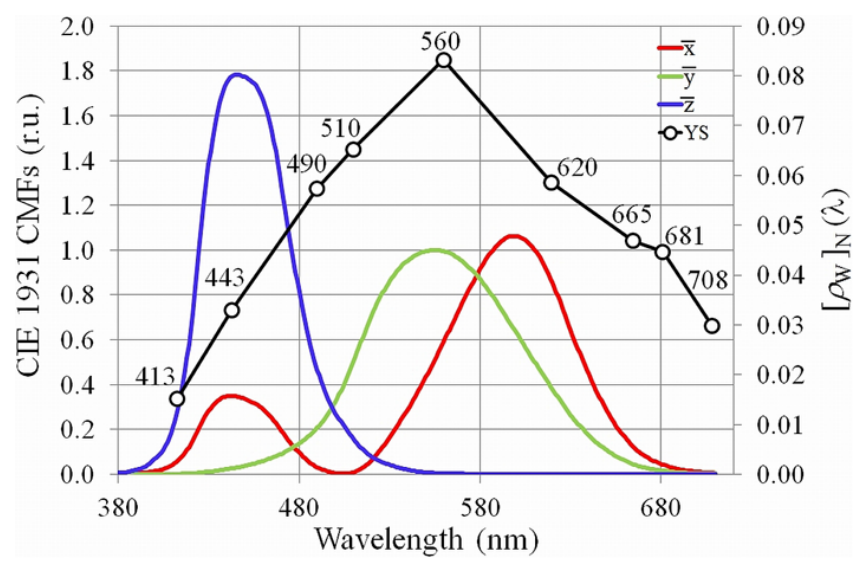

Fig. 1. The CIE $19312^{\circ}$ Colour Matching Functions for $\bar{x}$ (red), $\bar{y}$ (green) and $\bar{z}$ (blue) determined per nanometre. The black line shows a reconstructed spectrum (Yellow Sea) of MERIS reflectance measured at nine bands (open circles).

\subsection{The FUME algorithm}

The FUME algorithm converts the normalized water-leaving reflectance from nine MERIS bands into a discrete $F U$ number in three steps. Step 1: calculation of the tristimulus values $X, Y, Z$ by calculating the convolution of the colour-matching functions (CMFs) and the normalized water-leaving reflectance (CIE, 1932). Step 2: calculation of the $(x, y)$ chromaticity coordinates by the ratio of $X$ or $Y$ tristimulus values and the sum of the tristimulus values. Step 3: determination of the $F U$ scale number by comparison of calculated $(x, y)$ values to the unique chromaticity coordinates of the twenty-one $F U$ numbers.

Step 1: tristimulus values are the amounts of three primaries that specify a colour stimulus of the human eye (Wyszecky and Stilles, 2000) and are noted as $X, Y$ and $Z$ (CIE, 1932). The CIE 1931 standard colourimetric 2-degree CMFs $\bar{x}$ (red), $\bar{y}$ (green) and $\bar{z}$ (blue) are presented in Fig. 1. These serve as weighting functions for the determination of the tristimulus values of the MERIS normalized waterleaving reflectance $\left[\rho_{\mathrm{W}}\right]_{\mathrm{N}}$ by Eq. (2a), (b) and (c):
$X=\int\left[\rho_{\mathrm{W}}\right]_{\mathrm{N}}(\lambda) \bar{x}(\lambda) \mathrm{d} \lambda$

$Y=\int\left[\rho_{\mathrm{W}}\right]_{\mathrm{N}}(\lambda) \bar{y}(\lambda) \mathrm{d} \lambda$

$Z=\int\left[\rho_{\mathrm{W}}\right]_{\mathrm{N}}(\lambda) \bar{z}(\lambda) \mathrm{d} \lambda$

Because MERIS does not provide full-spectral coverage, the reflection spectrum is first reconstructed by linear interpolation between band $n=1(412.5 \mathrm{~nm})$ and band $n=9$ $(708 \mathrm{~nm})$ with a resolution of $1 \mathrm{~nm}$. An example is shown as a black line in Fig. 1. Note that the linear interpolation at $\lambda_{i}(\mathrm{~nm})$ is always carried out between subsequent bands $(n, n+1)$ with the condition $\left(\lambda_{n}<\lambda_{i}<\lambda_{n+1}\right)$. The tristimulus values for $X, Y$ and $Z$ are obtained by a Riemann sum approximation of the integrals with $\Delta \lambda=1 \mathrm{~nm}$ resolution:

$X=\sum_{i=413}^{708}\left[\rho_{\mathrm{W}}\right]_{\mathrm{N}}\left(\lambda_{i}\right) \bar{x}(\lambda) \Delta \lambda$

$Y=\sum_{i=413}^{708}\left[\rho_{\mathrm{W}}\right]_{\mathrm{N}}\left(\lambda_{i}\right) \bar{y}(\lambda) \Delta \lambda$

$Z=\sum_{i=413}^{708}\left[\rho_{\mathrm{W}}\right]_{\mathrm{N}}\left(\lambda_{i}\right) \bar{z}(\lambda) \Delta \lambda$.

Step 2: subsequently, the chromaticity coordinates $x, y$ and $z$ are calculated from the ratio of each of the tristimulus values and the sum of the values:

$x=\frac{X}{X+Y+Z} \quad y=\frac{Y}{X+Y+Z} \quad z=\frac{Z}{X+Y+Z}$.

As $x+y+z=1$, and therefore $z=1-x-y$, the third coordinate offers no additional information and only two coordinates (by convention $x$ and $y$ ) are used to represent the colour in a so-called chromaticity diagram (see, e.g. Mobley, 1994). The white point $W$ in the chromaticity diagram has the coordinates $x=y=z=1 / 3$ (Fig. 2). The ratio of the distance 
Table 3. Angles $\alpha_{i}$ of $F U$ number in degrees ( $i=1$ to 21 ) and the 20 boundary angles $\alpha_{i T}$ that are used in the discrete classification of ocean colour.

\begin{tabular}{llllllll}
\hline$i$ & $\alpha_{i}^{\circ}$ & $\alpha_{i T}^{\circ}$ & $\begin{array}{l}\text { If } \alpha_{\mathrm{M}}>\alpha_{i T} \\
\text { then } F U=\end{array}$ & $i$ & $\alpha_{i}^{\circ}$ & $\alpha_{i T}^{\circ}$ & $\begin{array}{l}\text { If } \alpha_{\mathrm{M}}>\alpha_{i T} \\
\text { then } F U=\end{array}$ \\
\hline 1 & 229.94 & 227.68 & 1 & 12 & 68.49 & 67.93 & 12 \\
2 & 225.41 & 219.27 & 2 & 13 & 67.36 & 65.98 & 13 \\
3 & 213.13 & 205.19 & 3 & 14 & 64.60 & 63.35 & 14 \\
4 & 197.25 & 189.20 & 4 & 15 & 62.11 & 60.37 & 15 \\
5 & 181.15 & 165.71 & 5 & 16 & 58.62 & 56.64 & 16 \\
6 & 150.26 & 133.96 & 6 & 17 & 54.65 & 52.09 & 17 \\
7 & 117.66 & 109.85 & 7 & 18 & 49.53 & 46.75 & 18 \\
8 & 102.05 & 95.14 & 8 & 19 & 43.96 & 41.82 & 19 \\
9 & 88.24 & 83.38 & 9 & 20 & 39.67 & 36.98 & 20 \\
10 & 78.53 & 74.62 & 10 & 21 & 34.28 & & 21 \\
11 & 70.71 & 69.60 & 11 & & & & \\
\hline
\end{tabular}

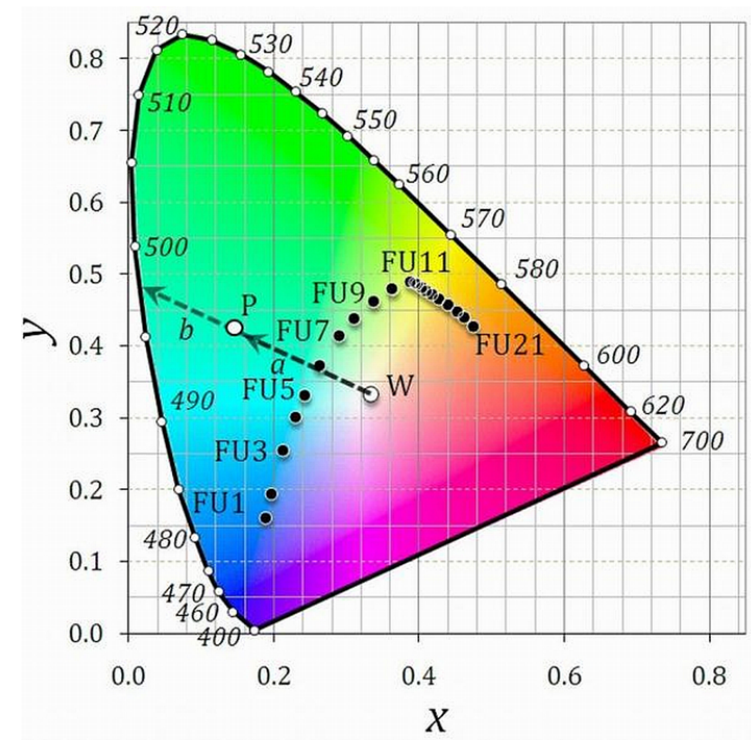

Fig. 2. The chromaticity coordinates, based upon transmission measurements, of the $F U$ scale colours 1 to 21 (black circles) and the white point $W$ (where $x_{\mathrm{W}}=y_{\mathrm{W}}=1 / 3$ ). The outer curved boundary is the spectral locus, with the corresponding monochromatic wavelengths shown in nanometres.

between $W$ and an arbitrary point $P(a)$ and the distance from $W$ to the spectral locus $(a+b)$, gives the colour saturation $(a /(a+b))$ or the intensity of the colour at $P$. In this way, the chromaticity coordinates $\left(x_{\mathrm{M}}, y_{\mathrm{M}}\right)$ for every MERIS pixel can be calculated.

Step 3: in the next step the $\left(x_{\mathrm{M}}, y_{\mathrm{M}}\right)$ is converted to a $F U$ number. The original $F U$ scale was created to make an objective classification of natural waters (see for a review Wernand and Gieskes, 2011). In 21 glass tubes a variable mixture of three standard solutions (distilled water, ammonia, copper sulphate, potassium chromate and cobalt sulphate) were created to obtain the colour palettes of the scale. These stan-

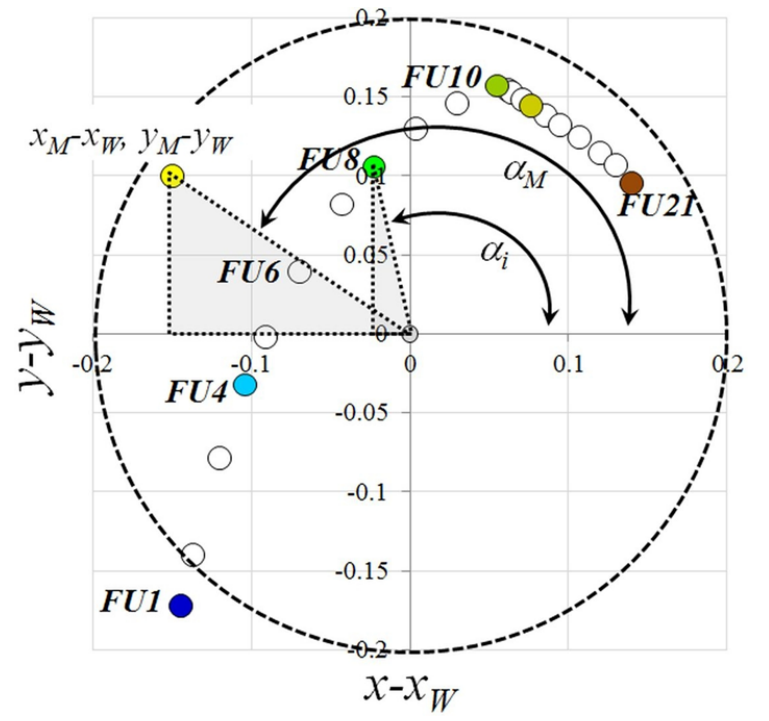

Fig. 3. Chromaticity diagram with scale colours FU1 to FU21 shown as dots, relative to the white point that is set at the origin. As an example the angle $\alpha_{i}\left(102.05^{\circ}\right.$, see Table 3$)$, determining the position of $F U 8$, is given.

dard solutions were recently reconstructed and their optical properties were measured in the laboratory with medium resolution spectrometers (Wernand and Van der Woerd, 2010a). The calculated chromaticity coordinates of the original $F U$ scale are presented in Table 2 and graphically shown as a line of black dots, between the white point and the locus, in the chromaticity diagram of Fig. 2. The FUME algorithm first shifts the origin to the white point $W$ with chromaticity coordinates $x_{\mathrm{W}}=y_{\mathrm{W}}=1 / 3$ (Fig. 3). Then it calculates the angle $\left(\alpha_{\mathrm{M}}\right)$ between the vector to a point with certain $F U$ coordinates $\left(x_{\mathrm{M}}, y_{\mathrm{M}}\right)$ and the positive $x$-axis (at $y-y_{\mathrm{W}}=0$ ), giving higher angles in an anticlockwise direction, and compares these with the angles $\left(\alpha_{i}\right)$ of the $F U$ solutions (Table 3 ). 


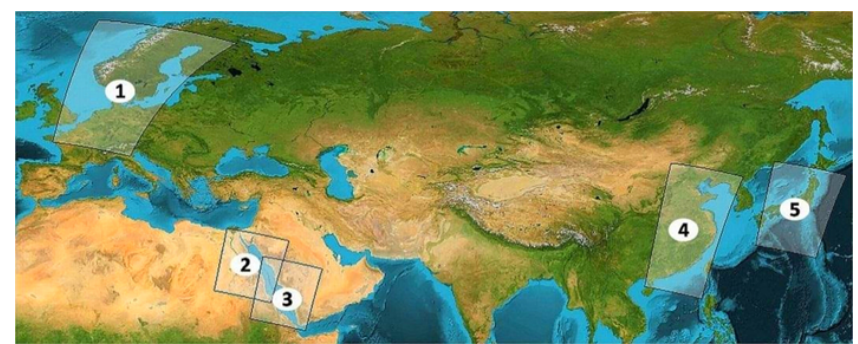

Fig. 4. Areas where MERIS data were extracted from ESA's MERCI database.

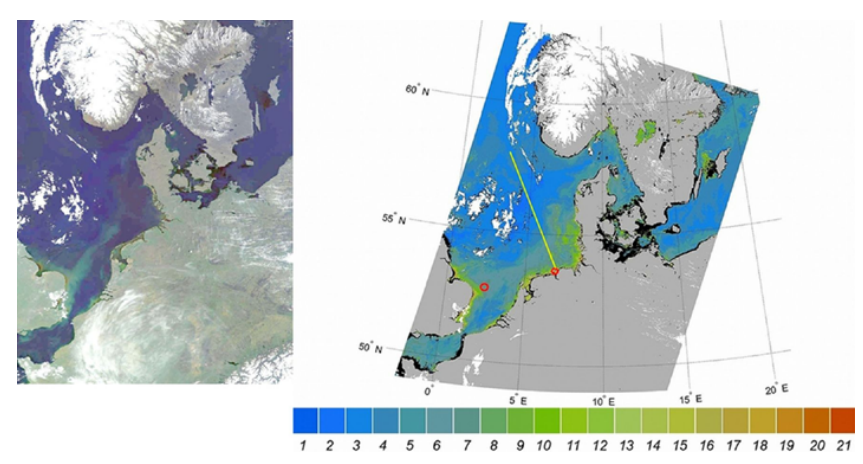

Fig. 5. Left: MERIS true colour image (4 May 2006). Right: the spring $F U$ map of the turbid North Sea shows $F U$ values ranging from 3 to 9 (red circle near England FU6). The Wadden Sea area within barrier islands north of Holland shows values between FU9 and $F U 18$. The area within the red circle indicates a pixel value of $F U=14$. The yellow line shows the transect extracted from the MERIS image and shown in Fig. 9.

All calculations were made with the atan 2 function (fourquadrant inverse tangent) and the derived angles (in radians) were multiplied by $180 / \pi$ to get the angles in degrees:

$\alpha_{\mathrm{M}}=\arctan \left(y_{\mathrm{M}}-y_{\mathrm{W}} x_{\mathrm{M}}-x_{\mathrm{W}}\right)$ modulus $2 \pi$.

Two examples are shown in Fig. 3. $\alpha_{i}$ is the angle matching FU8. The yellow dot is derived from the normalized spectral reflectance of a MERIS-pixel and coordinates $\left(x_{\mathrm{M}}-\right.$ $\left.x_{\mathrm{W}}=-0.15, y_{\mathrm{M}}-y_{\mathrm{W}}=0.1\right)$ and angle $\left(\alpha_{\mathrm{M}}\right)$. Finally, the boundaries distinguishing the various $F U$ numbers were defined. The colour transition angle $\alpha_{i T}$, under which a scale number transition takes place, was taken according to Eq. (6):

$\alpha_{i T}=\frac{\left(\alpha_{i}+\alpha_{i+1}\right)}{2}$.

Both $\alpha_{i}$ and $\alpha_{i T}$ are presented in Table 3. The $F U$ numbers for a given MERIS pixel $\mathrm{M}$ with chromaticity coordinates $x_{\mathrm{M}}-x_{\mathrm{W}}=-0.15$ and $y_{\mathrm{M}}-y_{\mathrm{W}}=0.1$ (yellow point in Fig. 3) can be determined as follows: first the angle (Eq. 5) is determined as $\alpha_{\mathrm{M}}=146^{\circ}$ and then is compared with a simple MATLAB loop for $i=1$ to 21 values of $\alpha_{i T}$ given in Table 3 . From this loop $\alpha_{\mathrm{M}}>\alpha_{i T}$ is true for the first time reaching the

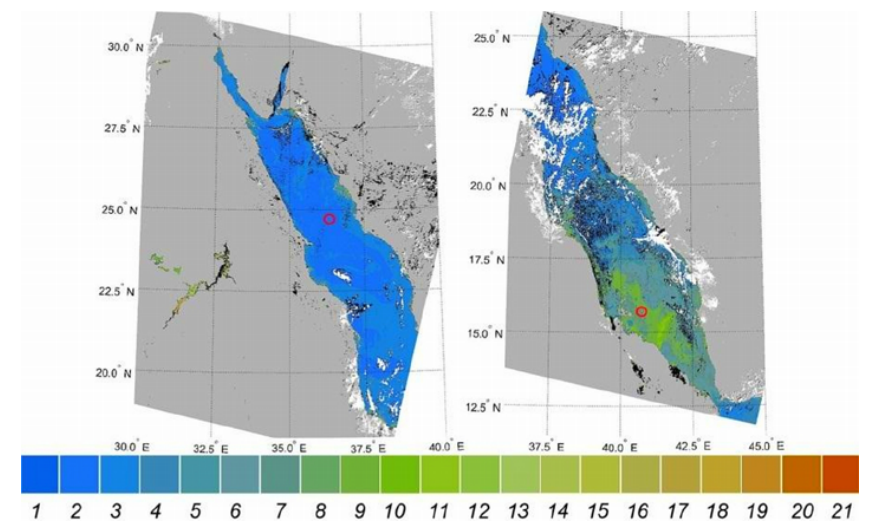

Fig. 6. The winter $F U$ map of the Red Sea, dated 22 (left) and 23 December 2003 (right) shows that open water of the northern part is mainly bluish $F U 1$ to $F U 2$ (in red circle $F U 2$ ), and near coast values are around $F U 3$. The southern part shows more greenish coloured water (in red circle $F U=8$ ).

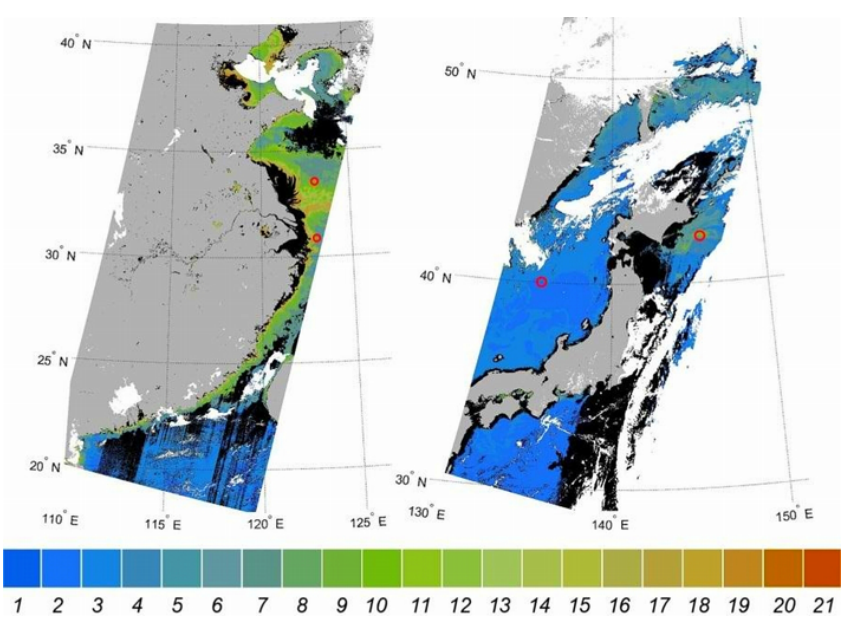

Fig. 7. The winter $F U$ map of the Yellow Sea, acquisition date 11 February 2009 (left), and the summer $F U$ map of the Sea of Japan, acquisition date 14 June 2004 (right). The Yellow Sea is mainly greenish brown with FU7 up to FU17 (FU9 in the lower red circle and $F U 11$ in the upper red circle). The Sea of Japan, a "blue sea", shows summer values of around FU2 to FU3 (in red circle $F U 2$ ). East of Hokkaido phytoplankton abundance greens the water to FU10 (FU9 within the red circle to the east).

angle $\alpha_{i T}=133.96$ degrees, which corresponds to a discrete value of $F U=6$ that is attributed to this MERIS pixel M.

\subsection{Ship-borne measurements}

The North Sea and the Wadden Sea (Hommersom et al., 2009) were optically sampled in 2006 (Fig. 5) and several lakes and rivers were sampled in 2001, 2006 and 2007. The surface radiance $L_{\mathrm{sfc}}$, sky radiance $L_{\text {sky }}$ and incoming solar irradiance $E_{\mathrm{S}}$ were measured simultaneously, using TRIOS hyper-spectral radiometers (Heuermann et al., 


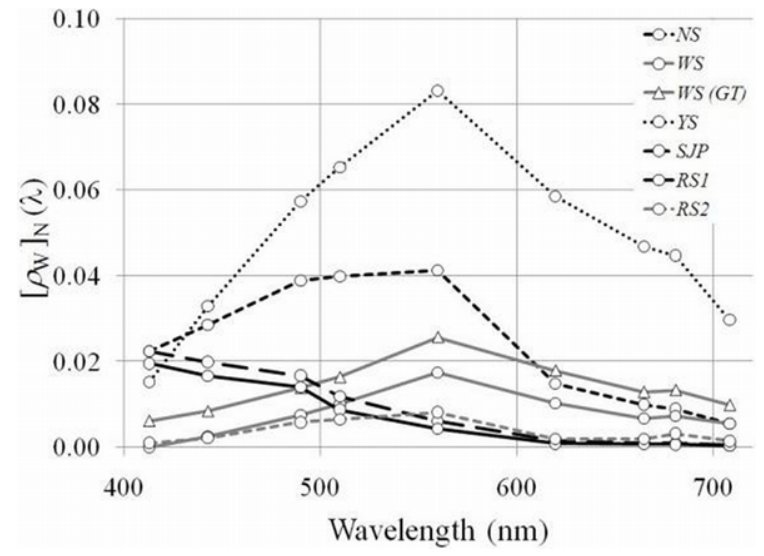

Fig. 8. Example of the pixel values of MERIS normalized waterleaving reflectance spectra of the North Sea (NS), Wadden Sea (WS), Yellow Sea (YS), Sea of Japan (SJP) and the northern and southern Red Sea (RS1 and RS2, respectively). Notice the similarity in the spectral shapes of the Wadden Sea spectra; WS is a MERIS normalized water-leaving reflectance spectrum and WS (GT) is the ground truth reflectance spectrum from ship-based measurements.

1999). Remote sensing reflectance was then calculated as $R_{\mathrm{RS}}=L_{\mathrm{w}} / E_{\mathrm{S}}$, where $L_{\mathrm{w}}$ is the water-leaving radiance (= $\left.L_{\mathrm{sfc}}-\rho L_{\mathrm{sky}}\right)$ and $E_{\mathrm{S}}$ is the downward irradiance just above the sea surface (Mueller et al., 2003). To a good approximation, $\left[\rho_{\mathrm{W}}\right]_{\mathrm{N}} \approx \pi R_{\mathrm{RS}}$ (Lee et al., 1994).

To illustrate the potential use of the satellite derived $F U$ maps, databases containing globally collected ship-borne $F U$ observations were consulted. From the oceanographic and meteorological database, archived by the United States National Oceanographic Data Centre (NOAA-NODC; Boyer et al., 2006), and from the ocean colour database at the Royal Netherlands Institute for Sea Research, $F U$ observations were extracted. To create the maps, $F U$ measurements were interpolated through an inverse distance weighted (IDW) technique (Watson and Philip, 1985) in an ARCGIS environment. The IDW interpolation was carried out over 2 degrees with a grid size of $0.2^{\circ}$.

\section{Datasets}

The FUME algorithm was applied to five MERIS images acquired over the areas shown in Fig. 4. These areas were chosen for their different sea colour properties (Wernand et al., 2013) and cover the North Sea (1), the Red Sea (2, 3), the Yellow Sea (4) and the Sea of Japan (5). The images were extracted from ESA's online database, the MERIS Catalogue and Inventory (MERCI, Brockman et al., 2005). MERIS Reduced Resolution (RR) geophysical products (Table 4) contain, among other products, a total of 14 spectral images of normalized band reflectances and the derived products for pigments (Algal-1, Algal-2, SPM and YS). A Reduced Resolution image has $4 \times 4$ less pixels than the
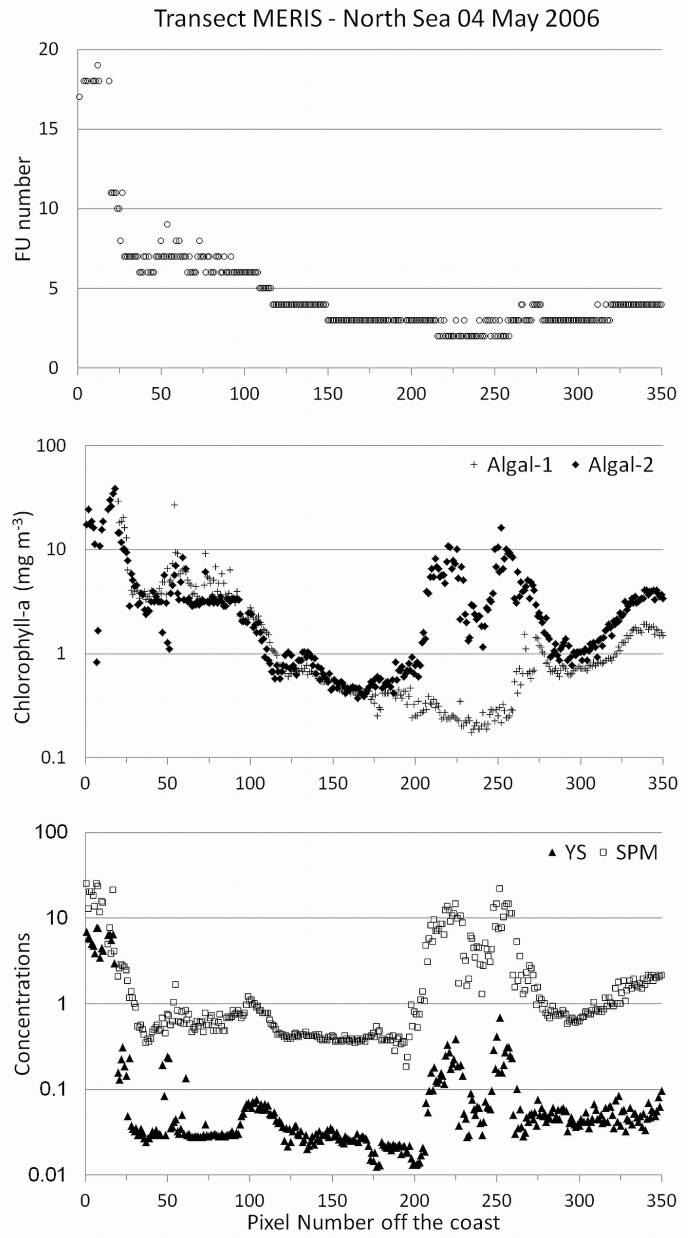

Fig. 9. Transect from the inner Wadden Sea to the central North Sea. The upper panel shows the $F U$ values, the middle panel shows the two MERIS products for chlorophyll $a$, and the lower panel shows the concentration of SPM (units in $\mathrm{g} \mathrm{m}^{-3}$ ) and yellow substance (absorption in $\mathrm{m}^{-1}$ at $442 \mathrm{~nm}$ ).

same image in Full-Resolution, thus representing an area of $1040 \mathrm{~m} \times 1160 \mathrm{~m}$.

To validate the FUME algorithm, a dataset of 53 simultaneously collected $F U$ observations and hyperspectral subsurface and above-water spectra was consulted. This dataset was established in 2001 and contains observations and optical data of a wide range of coloured water types, such as river, lake, coastal and open sea, with the $F U$ scale varying from FU3 (open sea) to FU21 (lakes). In addition, one dataset was included from Hommersom et al. (2009) that was made close ( $2.5 \mathrm{~h}$ prior) to the MERIS image acquisition time on 4 May 2006.

The routine collection of $F U$ measurements in all world seas was once very intense, mainly in the 20th century, and over 220000 measurements are known and available (Wernand et al., 2013). However, in the first decade of the 21st century the $F U$ data collection was much more limited and/or 
Table 4. Reference to the acquired MERIS Reduced Resolution images.

\begin{tabular}{llll}
\hline Sea & Area & Product name & Start time UTC \\
\hline North Sea & 1 & MER_RR_2PQBCM20060504 & 04-MAY-2006 10:11:24 \\
Red Sea north & 2 & MER_RR_2PQBCM20031222 & 22-DEC-2003 07:56:09 \\
Red Sea south & 3 & MER_RR_2PQBCM20031223 & 23-DEC-2003 07:26:02 \\
Yellow Sea & 4 & MER_RR_2PPBCM20090211 & 11-FEB-2009 02:27:39 \\
Sea of Japan & 5 & MER_RR_2PQBCM20040614 & 14-JUN-2004 01:07:24 \\
\hline
\end{tabular}

Table 5. RGB values for the reproduction of the $F U$ legend.

\begin{tabular}{llllllll}
\hline$F U$ no. & $\mathrm{R}$ & $\mathrm{G}$ & $\mathrm{B}$ & $F U$ no. & $\mathrm{R}$ & $\mathrm{G}$ & $\mathrm{B}$ \\
\hline 1 & 33 & 88 & 188 & 12 & 148 & 182 & 96 \\
2 & 49 & 109 & 197 & 13 & 165 & 188 & 118 \\
3 & 50 & 124 & 187 & 14 & 170 & 184 & 109 \\
4 & 75 & 128 & 160 & 15 & 173 & 181 & 95 \\
5 & 86 & 143 & 150 & 16 & 168 & 169 & 101 \\
6 & 109 & 146 & 152 & 17 & 174 & 159 & 92 \\
7 & 105 & 140 & 134 & 18 & 179 & 160 & 83 \\
8 & 117 & 158 & 114 & 19 & 175 & 138 & 68 \\
9 & 123 & 166 & 84 & 20 & 164 & 105 & 5 \\
10 & 125 & 174 & 56 & 21 & 161 & 77 & 4 \\
11 & 149 & 182 & 69 & & & & \\
\hline
\end{tabular}

has not yet been recorded in the central archives. Therefore, we have chosen to show data from the same seasons in earlier years. For the Red Sea, 52 observations are available and were collected during the winters of 1895 to 1898 . For the Yellow Sea, $2882 \mathrm{FU}$ observations were collected during the winters of 1930 to 1999.

\section{Results}

\subsection{MERIS $F U$ maps}

For all five MERIS images the reflectance values in bands 19 per pixel were converted to chromaticity coordinates and into $F U$ numbers using Eqs. (3) to (5). Converted images are further referred to as $F U$ maps. These $F U$ maps are presented in Figs. 5, 6 and 7. In these figures we have used the MERIS flags per pixel to identify land (grey), clouds (white) and the failure to collect observations or retrieve water-leaving reflectances (black). The legend (RGB - red, green, blue - values are given in Table 5) represents the $F U$ colours as close as possible. The $\left[\rho_{\mathrm{W}}\right]_{\mathrm{N}}$ spectral signatures at the locations marked with a red circle in the maps are plotted in Fig. 8.

The first $F U$ map shown in Fig. 5, acquisition date of 4 May 2006, covers the North Sea, the Baltic and the Wadden Sea. The colour of the North Sea varies between FU3 and FU9. The colour within the left red circle situated between the Thames and Humber estuaries was estimated as FU6. The central North Sea shows values of FU3 to FU4 with an oc-

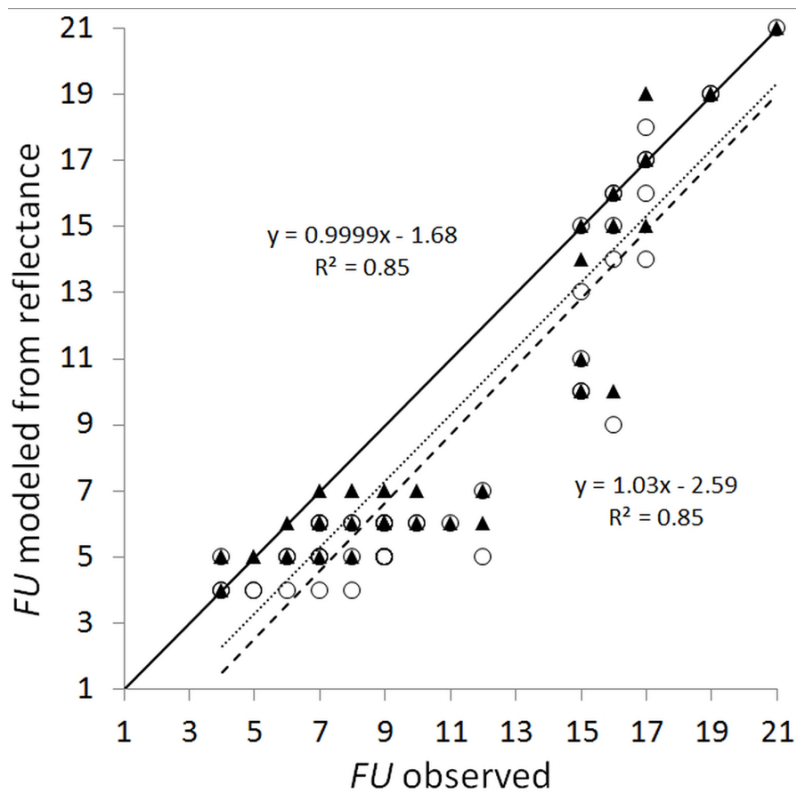

Fig. 10a. Scatter plot of the $F U$ numbers modelled using full reflectance spectra (black triangles) with trend line (dotted), and from the reflectance of MERIS bands (open circles) with trend line (intermittent), versus in situ observed $F U$ numbers. The full black line is the 1:1 line. See Fig. $10 \mathrm{~b}$ for the confusion matrix.

casional FU2 (very blue oceanic waters). The Wadden Sea, a large intertidal sea behind multiple barrier islands, north of Holland and Germany and west of Denmark, is dominated by sediment and outflow of humic-acid rich river water and has higher $F U$ values, up to $F U=18$.

Figure 6 shows a winter $F U$ map of both the northern and southern Red Sea taken on 22 and 23 December 2003, respectively. The colour of the northern Red Sea is mainly FU2 to FU3 with maximum values of FU5. The southern part, which is shallower than the northern part, shows a possible plankton bloom starting south of $17.5^{\circ} \mathrm{N}$ with values of $F U 8$ (red circle) to FU11. The water flowing through the narrow strait of Bab-al-Mandab into the Gulf of Aden (the area at the most south-eastern point on the map) shows much bluer values: $F U 2$ to $F U 4$. 


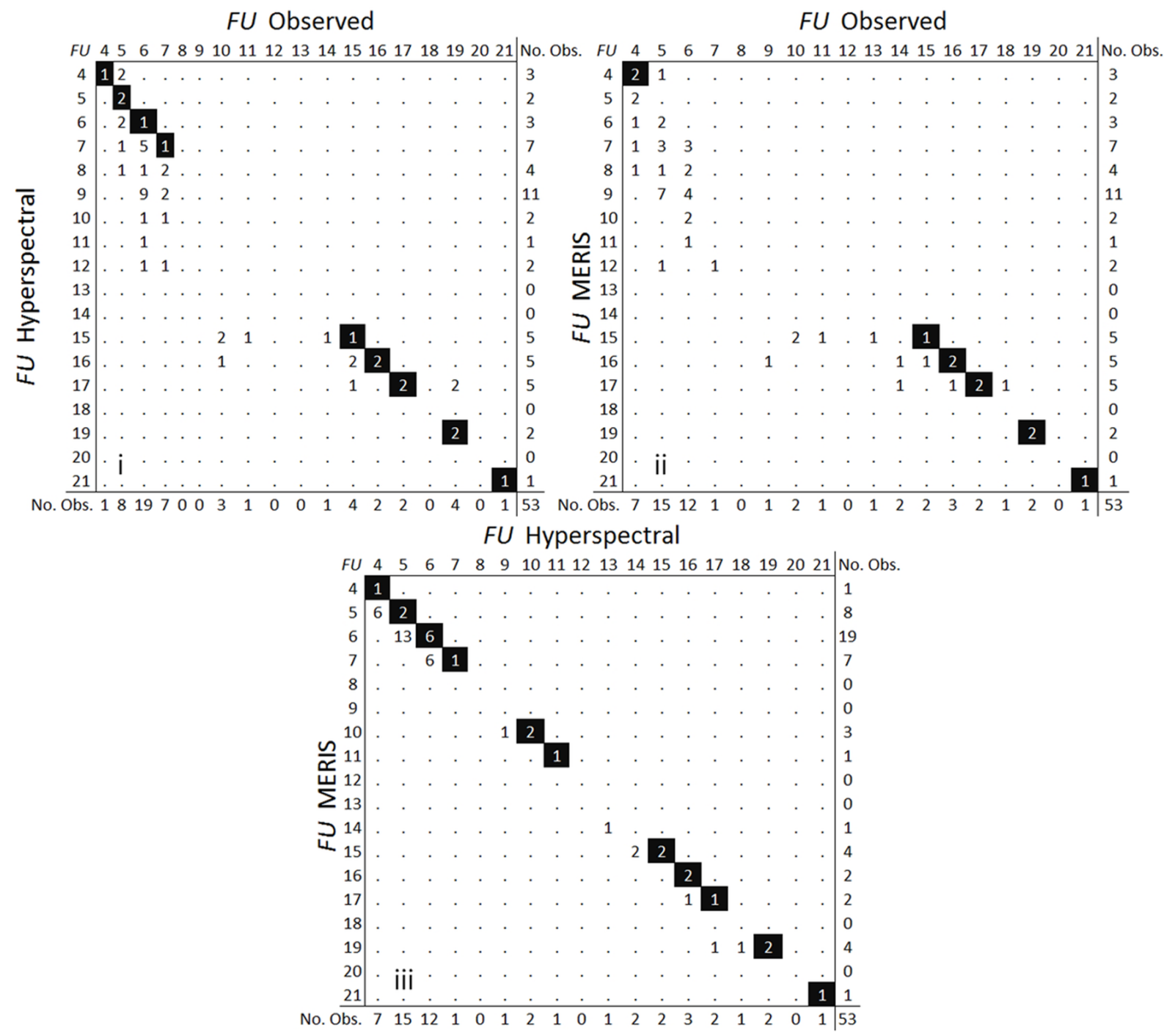

Fig. 10b. The confusion matrices of (i) $F U$ modelled hyperspectral data as a function of observed $F U$ number, (ii) $F U$ modelled MERIS data as a function of observed $F U$ number, and (iii) $F U$ modelled from MERIS spectral bands as a function of $F U$ modelled hyperspectral data.

Figure 7 shows $F U$ maps of the Yellow Sea (left) and the Sea of Japan (right) acquired on 11 February 2009 and 14 June 2004, respectively. The outflow of the Yangtze River (south of the red circle in the left image) shows high $F U$ values, between FU7 up to real brownish colours of FU19. Unfortunately, the area close to the river outflow is flagged as a "no data area". Within the red circle a value of FU9 is calculated. The Sea of Japan (right panel) shows values of $F U 2$ to FU3 (within the red circle the value is FU2). Remarkable is the relative green area east of Hokkaido (FU9 marked by the red circle east) with values up to FU10. To verify our results the MERIS Level 2 chlorophyll product was consulted, which showed high concentrations of chlorophyll $a\left(>2 \mathrm{mg} \mathrm{m}^{-3}\right)$ east of Hokkaido and concentrations between 0.1 and $0.5 \mathrm{mg} \mathrm{m}^{-3}$ in the Sea of Japan.

\subsection{Ground truth}

The reflection spectrum at the match-up station in the Wadden Sea (WS (GT)) is plotted in Fig. 8 and appears very similar in shape to the MERIS spectrum (WS). By extraction of the reflection at exactly all 9 MERIS bands and running the FUME algorithm, a value of $F U=15$ was retrieved. The MERIS pixel at this location (red circle in the Wadden Sea) has a calculated $F U$ value of 14 , which is in good agreement with the ground truth $F U$ value considering possible adjacency effects of tidal flats within the pixel.

The MERIS water-quality products and FUME results were extracted along a transect (yellow line in Fig. 5) perpendicular to the coast. The transect starts at the match-up point in the Wadden Sea (red circle) and ends in the central North Sea. The results are shown in the three panels of 


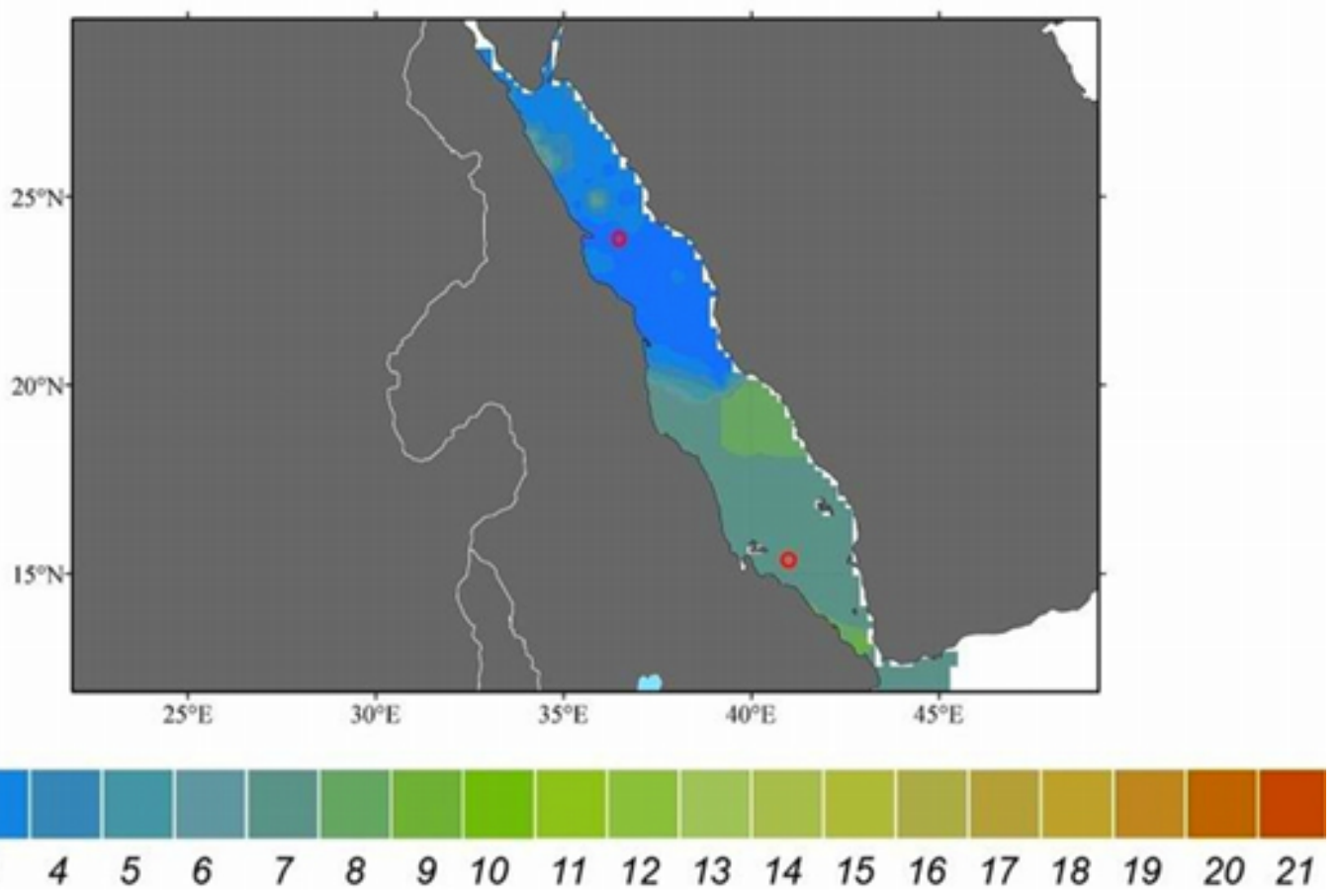

Fig. 11. The Red Sea map based on $52 F U$ in situ observations collected during the winters of 1895 to 1898 . Within the northern red circle $F U=2$ and within the southern red circle $F U=7$. This colour boundary can also be observed 100 yr later in the MERIS map of Fig. 6 .
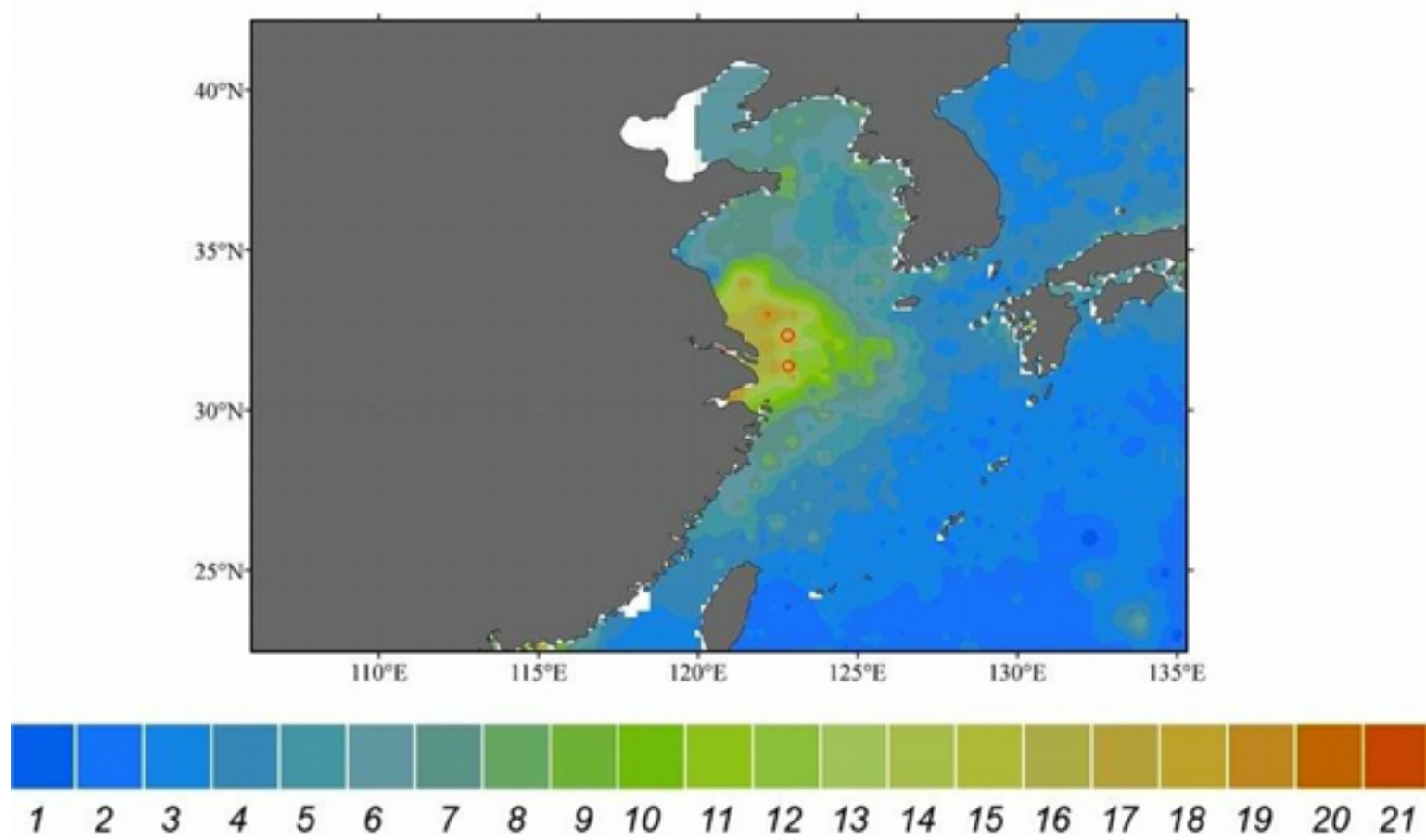

Fig. 12. Yellow Sea map based on $2882 F U$ in situ observations collected during winter between 1930 and 1999 . Within the red circles the colour is FU12 (lower, in front of the Yangtze outflow) and FU14 (upper). Near coast FU numbers are as high as 18 to 20. 
Fig. 9. Within the first 30 pixels the waters are within or close to the Wadden Sea, characterized by very high loads of sediment $\left(>1 \mathrm{~g} \mathrm{~m}^{-3}\right)$ and yellow substance (absorption $>1 \mathrm{~m}^{-1}$ at $442 \mathrm{~nm}$ ), corresponding to $F U$ values above 7 . In the next part (pixels 30-170), the $F U$ values show a gradual gradient from 7 to 3, reflecting a decrease in algal pigments (both Algal-1 and Algal-2 products) because both YS and SPM are rather constant in this interval. An interesting feature can be observed in the pixels (170-300) where the Case 2 water algorithm seems to fail (unrealistic high SPM and YS), likely due to additional scatter by cirrus clouds. Fortunately, the $F U$ scale seems robust and corresponds rather well with the Algal-1 product.

Based on field measurements, a comparison between observed and modelled $F U$ numbers was made (Fig. 10). The correlation between observed and modelled $F U$ numbers is around the 1:1 line (black line, Fig. 10a). To give additional insight into the results presented in Fig. 10a, confusion matrices were made from the rather sparse data and are presented in Fig. 10b. For modelled hyperspectral data as a function of observed $F U$ (Fig. 10b-i), $51 \%$ is within $1 F U$ scale number; for modelled MERIS data as a function of observed $F U$ (Fig. 10b-ii), $40 \%$ is within $1 F U$ scale number; and for the modelled MERIS data as a function of modelled hyperspectral data (Fig. 10b-iii), $98 \%$ is within $1 F U$ scale number. $F U$ numbers derived from the full spectrum and MERIS derived $F U$ correlate equally with in situ data $\left(R^{2}=0.85\right)$. The largest outliers were found in the 11-16 FU mid-range. These are the green-yellowish water colours, which corresponds to $\sim 500-600 \mathrm{~nm}$ visible light. The $11-16$ FU colours are very close in the chromaticity diagram; therefore, small errors in the modelled $F U$ could induce a wrong $F U$ index. This problem may explain part of the outliers of Fig. 10a.

Comparing the MERIS winter $F U$ maps of the northern and southern Red Sea in Fig. 6 with the winter IDW $F U$ map of Fig. 11, similar patterns can be recognized despite a time gap of over a century between data acquisition. When we compare these maps, it seems that the colour of the Red Sea did not change significantly over time, although we cannot say anything about intermediate colour changes between 1899 and 2002. Within the red circles, the MERIS FU map (Fig. 6) shows FU2 for the northern location and FU8 for the southern location, while the IDW $F U$ map gives identical values at both locations.

The $F U$ map for the Yellow Sea, based on $2882 F U$ in situ observations collected during the winters of 1930 to 1999, is shown in Fig. 12. The Yellow Sea shows $F U$ numbers of $F U 4$ in open sea areas to values of FU2O in front of the outflow of the Yangtze River. Both the MERIS map of Fig. 7 and the IDW interpolation of Fig. 12 show similar colour patterns. The red circles on the MERIS map indicate $F U 9$ in the lower and FU11 in the upper. In the IDW map, respectively, FU12 and FU14 are indicated. A possible explanation of the bluing of the water (bluer colours show up in the 2008/9 map) is the reduced outflow of Yangtze water into the Yellow Sea due to the hydroelectric Three Gorges Dam which became operational in 2003. The effect is a reduced upwelling and thus productivity, resulting in less green water (Chen, 2000; Gong et al., 2006).

\section{Discussion and conclusions}

In this paper an algorithm is presented that allows retrieval of the Forel-Ule sea colour from the MERIS satellite sensor. The Forel-Ule colour can be seen as the colour standard closest to the real colour of water. The elegance of our algorithm is that it converts multispectral observations to one simple number that is only dependent on a well-known universal set of colourimetric functions. The classification of sea water is simplified by means of a numerical value between 1 and 21, instead of a classification by a normalized waterleaving spectral reflectance signature or the concentrations of the dominant optical constituents.

The approach is demonstrated by the processing of multispectral observations of oceans and coastal waters made by the MERIS ocean colour sensor to $F U$ maps that cover colour classes between indigo blue, green and brown. Five different seas were selected worldwide; these were processed to obtain $F U$ maps. The maps show very detailed patterns and gradients, mainly in the near coastal zones as expected by the more pronounced hydrographical gradients there. When the MERIS maps of sea and ocean colour distribution were compared with ground truth Forel-Ule observations mapped in the same season, similar patterns and $F U$ numbers were observed, even when $F U$ numbers of more than a century ago were processed. This opens new ways to study the spatial and temporal evolution of the colour of the sea worldwide. The FUME algorithm can easily be adapted to data from other satellites that have enough bands in the visible part of the spectrum to properly derive the colour of the water.

Acknowledgements. Marieke Eleveld, Steef Peters and Reinold Pasterkamp from the Institute for Environmental Studies, Free University, Amsterdam, are thanked for their initiative to develop MATLAB routines that were used and adapted to generate satellite derived Forel-Ule maps. Menno Regeling is thanked for the ForelUle data collection in the North Sea (June 2001). Thanks are due to W. Gieskes (Dept. Ocean Ecosystems, University of Groningen, the Netherlands) for discussions and comments. MERIS data has been provided by the European Space Agency (ESA). We would like to thank J. Piera Fernandez for his suggestions to improve Fig. 3 and Fig. 10.

Edited by: O. Zielinski 


\section{References}

Antoine, D., Morel, A., Gordon, H. R., Banzon, V. F., and Evans, R. H.: Bridging ocean colour observations of the 1980s and 2000s in search of long-term trends, J. Geophys. Res. 110, C06009, doi:10.1029/2004JC002620, 2005.

Behrenfeld, M. J., O’Malley, R. T., Siegel, D. A., McClain, C. R., Sarmiento, J. L., Feldman, G. C., Milligan, A. J., Falkowski, P. G., Letelier, R. M., and Boss, E. S.: Climate-driven trends in contemporary ocean productivity, Nature, 444, 752-755, 2006.

Boyer, T. P., Antonov, J. I., Garcia, H. E., Johnson, D. R., Locarnini, R. A., Mishonov, A. V., Pitcher, M. T., Baranova, O. K., and Smolyar, I. V.: World Ocean Database 2005, edited by: Levitus, S., NOAA Atlas NESDIS 60, US Government Printing Office, Washington, DC, 190 pp., 2006.

Brockmann, C., Block, T., Faber, O., Fokken, L., and Kock, O.: MERCI - MERIS Catalogue and Inventory, MERIS-ATSR workshop, ESA, ESRIN, 2005.

Chen, C.-T. A.: The Three Gorges dam: Reducing the upwelling and thus productivity in the East China Sea, Geophys. Res. Lett., 27, 381-383, 2000.

CIE: Commission Internationale de l'Éclairage proceedings, 1931, Cambridge University Press, Cambridge, 1932.

ESA: MERIS Algorithm Theoretical Basis Documents, http: //envisat.esa.int/instruments/meris/pdf/ (last access: 7 January 2012), 2012.

Forel, F. A.: Une nouvelle forme de la gamme de couleur pour l'étude de l'eau des lacs, Archives des Sciences Physiques et Naturelles/Société de Physique et d'Histoire Naturelle de Genève, VI, 25 pp., 1890.

Gong, G.-C., Chang, J., Chiang, K.-P., Hsiung, T.-M., Hung, C.-C., Duan, S.-W., and Codispoti, L. A.: Reduction of primary production and changing of nutrient ratio in the East China Sea: Effect of the Three Gorges Dam? Geophys. Res. Lett., 33, L14609, doi:10.1029/2006GL025800, 2006.

Gordon, H. R. and Voss, K. J.: MODIS Normalized Water-leaving Radiance Algorithm Theoretical Basis Document, Vol. MOD 18, Version 4, 1999.

Heuermann, R., Reuter, R., and Willkomm, R.: RAMSES, A modular multispectral radiometer for light measurements in the UV and VIS, in: SPIE Proc. Series, 3821, 279-285, 1999.

Hommersom, A., Peters, S., Wernand, M. R., and De Boer, J.: Spatial and temporal variability in bio-optical properties of the Wadden Sea, Estuarine, Coastal and Shelf Sci., 83, 360-370, 2009.

Hommersom, A., Wernand, M. R., Peters, S., Eleveld, M. A., van der woerd Woerd, H. J., and de Boer, J.: Spectra of a shallow sea - unmixing for class identification and monitoring of coastal waters. Ocean Dynamics, 61, 463-480, doi:10.1007/s10236-0100373-4, 2011.

IOCCG: Minimum Requirement for an Operational Ocean-Colour Sensor for the Open Ocean, Report Number 1, http://www.ioccg. org/reports_ioccg.html (last access: 7 January 2012), 1998.

Lee, Z. P., Carder, K. L., Hawes, S. K., Steward, R. G., Peacock, T. G., and Davis, C. O.: Model for the interpretation of hyperspectral remote-sensing reflectance, Appl. Opt., 33, 5721-5732, 1994.
Mobley, C. D.: Light and Water: Radiative Transfer in Natural Waters, Academic Press, San Diego, 1994.

Moore, T. S., Campbell, J. W., and Dowell, M. D.: A class-based approach to characterizing and mapping the uncertainty of the MODIS ocean chlorophyll product, Remote Sens. Environ., 113, 2424-2430, 2009.

Morel, A. and Prieur, L.: Analysis of variations in ocean color, Limnol. Oceanogr., 22, 709-722, 1977.

Mueller, J. L., Davis, C., Arnone, R., Frouin, R., Carder, K., Lee, Z. P., Steward, R. G., Hooker, S., Mobley, C. D., and McLean, S.: Above-Water Radiance and Remote Sensing Reflectance Measurement and Analysis Protocols. Ocean Optics Protocols for Satellite Ocean Colour Sensor Validation, Revision 4, Volume III: Radiometric Measurements and Data Analysis Protocols, NASA/TM-2003-21621/Rev4 III, 21-31, 2003.

Odermat, D., Gitelson, A., Brando, V. E., and Schaepman, M.: Review of constituent retrieval in optically deep and complex waters from satellite imagery, Remote Sens. Environ., 118, 116126, 2012.

Polovina J. J., Howell, E. A., and Abecassis, M.: Ocean's least productive waters are expanding, Geophys Res. Let., 35, L03618, doi:10.1029/2007GL031745, 2008.

Rast, M., Bezy, J. L., and Bruzzi, S.: The ESA Medium Resolution Imaging Spectrometer MERIS: a review of the instrument and its mission, Int. J. Remote Sens., 20, 1681-1702, 1999.

Tilstone, G., Peters, S. W. M., Van der Woerd, H., Eleveld, M., Ruddick, K, Schoenfeld, W., Krasemann, H., Martinez-Vicente, V., Blondeau-Patissier, D., Röttgers, R., Sørensen, K., Jorgensen, P., and Shutler, J.: Variability in specific-absorption properties and their use in a semi-analytical Ocean Colour algorithm for MERIS in North Sea and Western English Channel Coastal Waters, Remote Sens. Environ., 118, 320-338, 2012.

Ule, W.: Die bestimmung der Wasserfarbe in den Seen, Kleinere Mittheilungen, A. Petermanns Mittheilungen aus Justus Perthes geographischer Anstalt, Gotha, Justus Perthes, 70-71, 1892.

Van der Woerd, H. J. and Pasterkamp, R.: HYDROPT: A fast and flexible method to retrieve chlorophyll-a from multispectral satellite observations of optically complex coastal waters, Remote Sens. Environ., 112, 1795-1807, 2008.

Watson, D. F. and Philip, G. M.: A Refinement of Inverse Distance Weighted Interpolation, Geoprocessing, 2, 315-327, 1985.

Wernand, M. R. and Gieskes, W. W. W.: Ocean optics from 1600 (Hudson) to 1930 (Raman); Shift in interpretation of natural water colouring, Union des océanographes de France, 2011.

Wernand, M. R. and Van der Woerd, H. J.: Spectral analysis of the Forel-Ule Ocean colour comparator scale, J. Europ. Opt. Soc. Rap. Public., 5, 10014s, doi:10.2971/jeos.2010.10014s, 2010a.

Wernand, M. R. and Van der Woerd, H. J.: Ocean colour changes in the North Pacific since 1930, J. Europ. Opt. Soc. Rap. Public., 5, 10015s, doi:10.2971/jeos.2010.10015s, 2010 b.

Wernand, M. R., van der Woerd, H. J., and Gieskes, W. W. W.: Trends in ocean colour and chlorophyll concentration from 1889 to 2000, worldwide, PLOS ONE, accepted, 2013.

Wyszecki, G. G. and Stilles, W. S.: Colour science; concepts and methods, quantitative data and formulae. Second edition, Wiley classics Library edition, Wiley \& Sons, INC, 127-129, 2000. 